\section{Spurious correlations in research on the effects of specific cognitive abilities}

\author{
Kimmo Sorjonen \& Bo Melin
}

Department of Clinical Neuroscience,

Karolinska Institutet, Stockholm, Sweden

\section{Abstract}

Studies on the effect of non- $g$ ability residuals have often employed double adjustment for general cognitive ability $(g)$, as they have calculated the ability residuals adjusting for $g$ and then calculated the effect of the non- $g$ residuals while adjusting for $g$. The present simulations demonstrate that the double adjustments may result in spurious negative associations between the non- $g$ residual on one cognitive ability, e.g. verbal ability, and variables with a positive association with another ability, e.g. SAT math and math ability. In analyses of the 1997 National Longitudinal Survey of Youth (NLSY97), the negative associations between non- $g$ residuals on verbal and math ability and aptitude test scores on the other ability vanished when not double adjusting for $g$. This indicates that the observed negative associations may be spurious and not due to differential investment of time and effort in one ability at the expense of the other ability, as suggested in the literature. Researchers of the effects of specific abilities are recommended to validate their findings and interpretations with analyses not double adjusting for $g$.

Keywords: double adjustment, general cognitive ability, non- $g$ residuals, spurious correlations, verbal and math abilities

\section{Introduction}

Two friends, A and B, spend the day at the market, selling apples, tomatoes, and potatoes at the same set price. In the evening it turns out that they have sold for exactly the same amount of money, but that A has sold more apples. They both suspect that A should, consequently, have sold less tomatoes or potatoes. A week later A and B participate in a psychological study where they take an intelligence test, measuring verbal, mathematical, technical as well as general ability. Afterwards it turns out that they received exactly the same general ability score, but that A got a higher verbal ability score. They both suspect that A should, consequently, have received a lower mathematical or technical ability score.

In general terms, if $\mathrm{Y}=\mathrm{X}+\mathrm{Z}$, then $\mathrm{Z}$ must decrease if $\mathrm{X}$ increases in order for $\mathrm{Y}$ to remain constant. This is a probable explanation of why residuals on lower order ability factors can be negatively correlated when partialling out variance due to higher order factors, e.g. general ability $(g)$ (Calvin et al., 2010; Gignac, 2016; Johnson \& Bouchard, 2007; Lynn, 1987). If somebody has a higher value than expected (i.e. a positive residual) on ability A, as predicted by $g$, then this must be "compensated" by a lower value than expected (i.e. a negative residual) on some other ability. Without this compensation, the individual's $g$ would have been predicted to be higher (Ashton \& Vernon, 1995; Nyborg \& Sommerlund, 1992; Vernon, 1990). Consequently, there does not seem to be any compelling reasons to assume that negative correlations between residuals are due to differential investment of time and effort in one ability at the expense of other abilities, as suggested by Coyle (2018a).

Ability tilt refers to a within-individual difference between standardized ability scores, e.g. math ability - verbal ability. Coyle and colleagues have demonstrated that ability tilts tend to correlate with other measures of the constituent variables, e.g. that the math ability - verbal ability difference tends to correlate positively with other measures of math ability than the one used for calculating the tilt and negatively with other measures of verbal ability, and vice versa for the verbal ability - math ability difference (Coyle, 2016; Coyle et al., 2014; Coyle, Snyder, \& Richmond, 2015). Coyle and colleagues have suggested that such tilt-correlations are due to differential investment of time and effort in one of the abilities at the expense of the other ability (Coyle, 2016, 2019, 2020, 2021; Coyle et al., 2014; Coyle, Snyder, \& Richmond, 2015; Coyle \& Greiff, 2021). However, recently it was shown that observed tilt-correlations may be spurious and due to the simple fact that two different measures of the same or similar abilities tend to correlate more positively than two measures of different abilities (Sorjonen et al., 2022).

Coyle and colleagues have also demonstrated positive (negative) associations between the residuals on ability or performance test scores, when removing variance explainable by $g$, and other similar (dissimilar) ability or performance test scores (Coyle, 2018b; Coyle et al., 2013; Coyle, Snyder, Richmond, et al., 2015; Coyle \& Pillow, 2008). For example, Coyle (2018b) found a positive association between math non- $g$ residuals and math aptitude test scores and a negative association between math non- $g$ residuals and verbal aptitude test scores, and vice versa for verbal non- $g$ residuals. Coyle and colleagues have suggested, similarly as the explanation of tilt-correlations above, that such correlations involving non- $g$ residuals are due to differential investment in one of the abilities at the expense of the other ability (Coyle, 2014, 2018a, 2018b; Coyle et al., 2013; Coyle, Snyder, Richmond, et al., 2015).

However, Coyle and colleagues (e.g. Coyle, $2018 \mathrm{~b}$ ) seem to have calculated the associations 
between non- $g$ residuals on ability or performance test scores and other ability or performance test scores while adjusting for $g$. This means that they have employed double adjustment for $g$, where the residuals have been calculated adjusting for $g$ and then the effect of the non- $g$ residuals on the outcome variable has been calculated while adjusting for $g$. In accordance with the thoughts presented above, we suspected that a negative $g$-adjusted association between, for example, the non- $g$ residuals on one ability and the aptitude test scores on another ability may be due to confounding by this other ability. For example, those with a higher (more positive) non- $g$ residual on math ability may tend to have a lower verbal ability compared with those with the same $g$ but with a lower (more negative) non- $g$ residual on math ability. Hence, a negative $g$-adjusted association between non- $g$ residuals on math ability and verbal aptitude test scores may be spurious and due to a confounding influence of verbal ability rather than a true independent association. The objective of the present study was to evaluate this hypothesis, employing both simulations and analyses of the same empirical data as used by Coyle (2018b).

\section{Method}

\section{Simulations}

Data generation. Data were generated through the following steps (upper part in Figure 1): (1) Virtual participants $(N=10,000)$ were allocated a true $g$ score from a random normal distribution $(M=$ $0, S D=1)$; (2) The participants were allocated true verbal, math, and tech ability scores from random normal distributions with a defined population correlation of 0.8 with the true $g$ score; (3) The participants were allocated five test scores for each of the three abilities from random normal distributions (only three test scores are shown in Figure 1). The population correlations between the ability and test scores ( $\lambda$ in Figure 1$)$ were drawn from a random uniform distribution between 0.5 and 0.9. The fourth step had two possibilities: (4a) The true verbal score was regressed on the true $g$ score and the true non- $g$ verbal residuals were calculated $\left(\varepsilon_{\mathrm{tv}}\right.$ in Figure 1). The participants were allocated a score on an outcome variable from a random normal distribution with a defined population correlation with the true non- $g$ verbal residual; (4b) The respondents were allocated a score on an outcome variable from a random normal distribution with a defined population correlation with the true math score. All values between -1 and 1 in steps of 0.01 , i.e. 201 simulations, were used as the defined population correlation in steps $4 \mathrm{a}$ and $4 \mathrm{~b}$. When using alternative $4 \mathrm{a}$ the outcome variable had a true independent association with the non- $g$ verbal residual while this was not the case when using alternative $4 \mathrm{~b}$. In the latter case an association between observed non- $g$ residuals and the outcome variable would be spurious.

Statistical analyses. A model with one second order factor and three first order factors with five indicators each was fitted to data (bottom part in Figure 1). The observed non- $g$ verbal residuals were calculated ( $\varepsilon_{\mathrm{ov}}$ in Figure 1). The outcome variable

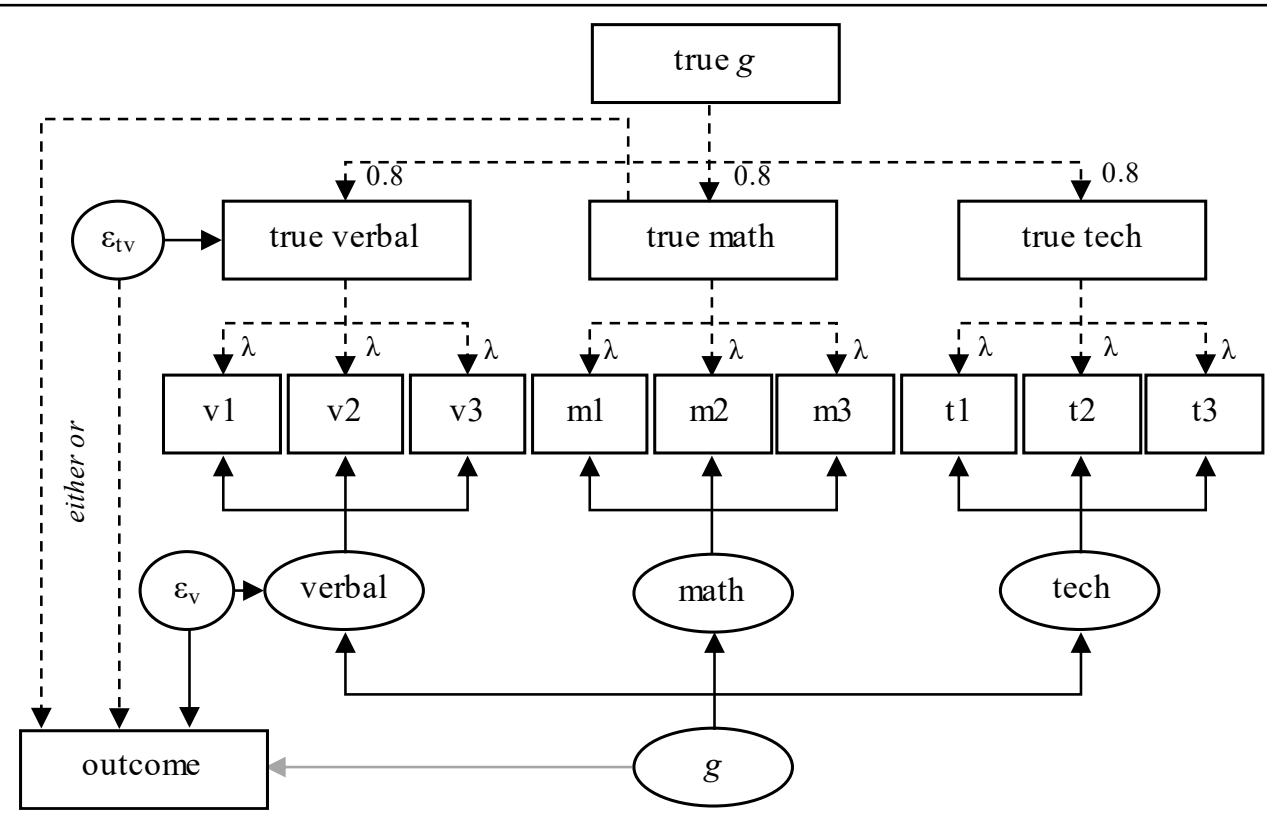

Figure 1. Steps in the data generation (dashed arrows) and analyzed model (solid arrows). The outcome variable was generated to have an independent association either with the true non- $g$ verbal residual ( $\varepsilon_{\text {tv }}$ ) or with true math ability, but not with both at the same time. The effect of estimated $g$ on the outcome variable (gray arrow) was either included or not included in the analyzed model. The effect of observed non- $g$ verbal residual ( $\left.\varepsilon_{\mathrm{ov}}\right)$ on the outcome variable was the main dependent variable in the analyses. Note: Five indicators of each of the three abilities were used in the actual simulations. 
was regressed either only on the observed non- $g$ verbal residuals or on both the observed non- $g$ residuals and the calculated $g$ score. The effect of the observed non- $g$ verbal residuals on the outcome variable and how this effect changed when the outcome had and did not have a true independent association with non- $g$ verbal residuals and when adjusting and not adjusting for calculated $g$ was the focus of interest in the analyses.

\section{Empirical analyses}

Participants. The sample consisted of 8984 participants (4385 women and 4599 men) of US youth born between 1980 and 1984 who participated in the 1997 National Longitudinal Survey of Youth (NLSY97). The dataset is available from the US Bureau of Labour Statistics (https://www.nlsinfo.org/content/cohorts/nlsy97).

Measurements. In 1997-1998 most of the participants (7008 with complete data) took 12 Armed Services Vocational Aptitude Battery (ASVAB) tests in: (1) general science (GS); (2) arithmetic reasoning (AR); (3) word knowledge (WK); (4) paragraph comprehension (PC); (5) numerical operations (NO); (6) coding speed (CS); (7) auto information (AI); (8) shop information (SI); (9) mathematical knowledge (MK); (10) mechanical comprehension (MC); (11) electronics information (EI); (12) assembling objects (AO). Data on the math and verbal subtests of the three college aptitude tests SAT, ACT, and PSAT were available for between 1003 and 1407 participants.

Statistical analyses. The analyzed models included one second order and four first order factors as well as an outcome variable (Figure 2). Following Coyle (2018b), we allowed the residuals of eight pairs of indicators to correlate. In one model the effect of non- $g$ verbal residual on the outcome variable was calculated, and in the other model the effect of non- $g$ math residual. The effects of the non$g$ residuals were calculated either adjusting or not adjusting for estimated $g$. Separate analyses were conducted with SAT math, SAT verbal, ACT math, ACT verbal, PSAT math, and PSAT verbal as the outcome variable, respectively. The estimations were conducted with robust (Huber-White) standard errors and missing values were handled by using full information maximum likelihood estimations.

Simulations and analyses were conducted with $\mathrm{R}$ 4.1.0 statistical software ( $\mathrm{R}$ Core Team, 2021) employing the lavaan package (Rosseel, 2012). The script and data are available at Open Science Framework at https://osf.io/f8buw/.

\section{Results}

\section{Simulations}

Results from the simulations are presented in Figure 3. We see that when the outcome variable was truly a function of the non- $g$ residual on verbal ability (lines with squares), the standardized regression effect of observed non- $g$ verbal residual on the outcome was the same as the true association and, importantly, was not affected by adjustment for $g$ (the gray and the black line with squares are identical).

We see something very different when the outcome variable was a function of true math ability, with no independent association with the true non- $g$ verbal residual (lines with triangles). Here, the standardized regression effect of observed non- $g$ verbal residual on the outcome was more negative when adjusting (gray line with triangles) compared with when not adjusting (black line with triangles)

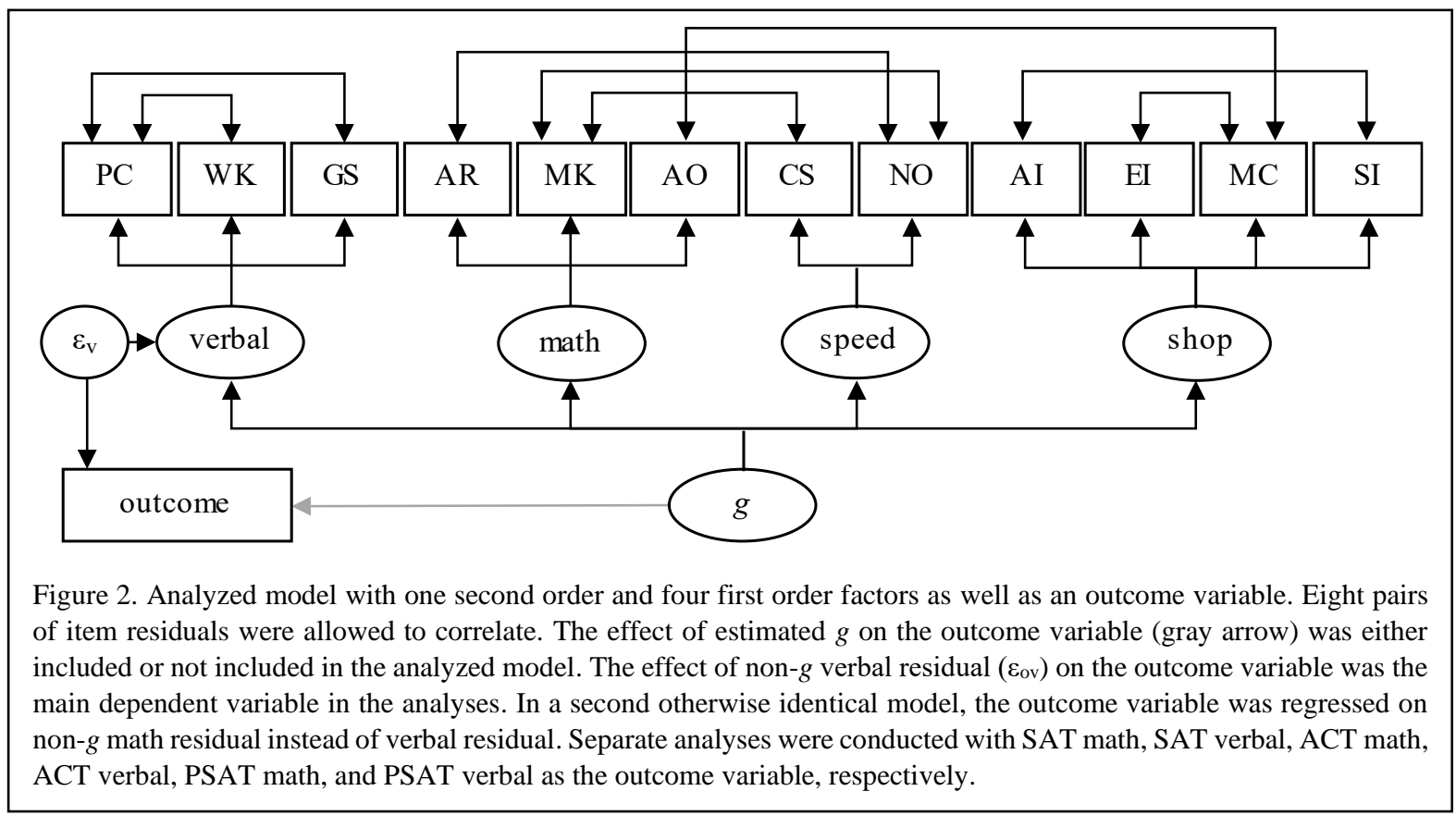




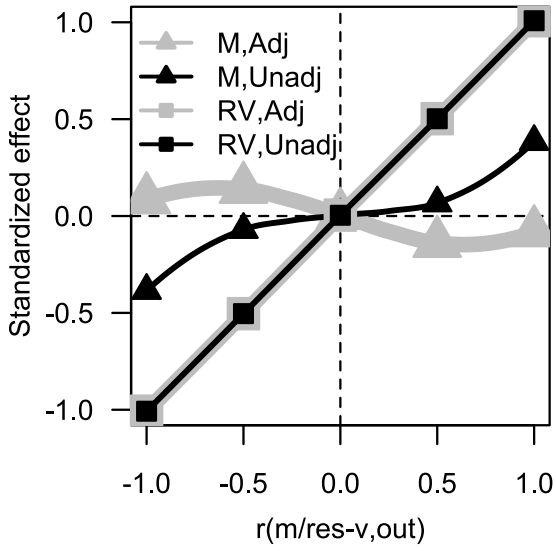

Figure 3. Predicted standardized effect of observed verbal non- $g$ residual on the outcome variable as a function of the correlation between true math score / true verbal non- $g$ residual and the outcome variable. Separately for situations when the outcome variable has been generated to have a true correlation with the verbal non- $g$ residual (squares) and when it has been generated to have a correlation with true math ability (i.e. no true independent association with the verbal non- $g$ residual, triangles). Also separately for situations when the effect of observed verbal non- $g$ residual on the outcome variable has (gray lines and markers) and has not (black lines and markers) been adjusted for $g$. Data simulated as illustrated in Figure 1.

for $g$, if the correlation between true math ability and the outcome was positive (to the right of the vertical dashed line) and vice versa if the correlation was negative (to the left of the vertical dashed line).

Based on the findings from these simulations we can make predictions what to expect when analyzing empirical data. If an observed effect of non- $g$ residual on one ability on an outcome variable is due to a true independent association, the effect should not change, at least not substantially, when adjusting or not adjusting for $g$. If the effect of non- $g$ residual changes when adjusting or not adjusting for $g$, the effect seems to be spurious and possibly due to a negative association between the non- $g$ residual on one ability and the score on another ability which, in turn, has a positive association with the outcome.

\section{Empirical analyses}

Results from the analyses of empirical data are presented in Figure 4. We see that when adjusting for $g$, verbal non- $g$ residuals (black triangles) had a negative effect on math aptitude test scores and math non- $g$ residuals (black squares) had a negative effect on verbal aptitude test scores. However, when not adjusting for $g$, these effects became more positive, i.e. weaker, and non-significant (zero included in the 95\% CI, white triangles and squares, respectively). Based on the findings in the simulations above, this pattern of effects suggests that the observed negative effects of the non- $g$ residual on verbal and math ability on aptitude test scores on the other ability are spurious and not due to true independent associations.

\section{Discussion}

The present study set out to investigate if negative $g$-adjusted associations between non- $g$ residuals on a specific ability and aptitude test scores on another ability may be spurious rather than due to a true independent association.

In accordance with Coyle (2018b), we found a negative effect of non- $g$ residual math ability on verbal aptitude test scores and a negative effect of non- $g$ residual verbal ability on math aptitude test scores when adjusting for $g$ in the NLSY97 dataset. Coyle and colleagues have proposed that negative effects of non- $g$ residual abilities are due to differential investment of time and effort in one ability at the expense of the other ability (Coyle, 2014, 2018a, 2018b; Coyle et al., 2013; Coyle, Snyder, Richmond, et al., 2015). However, the negative effects of the non- $g$ residuals became more positive, i.e. weaker, and non-significant when not adjusting for $g$. This change in effects corresponds to what happened in the simulations when the outcome variable was generated to have a positive

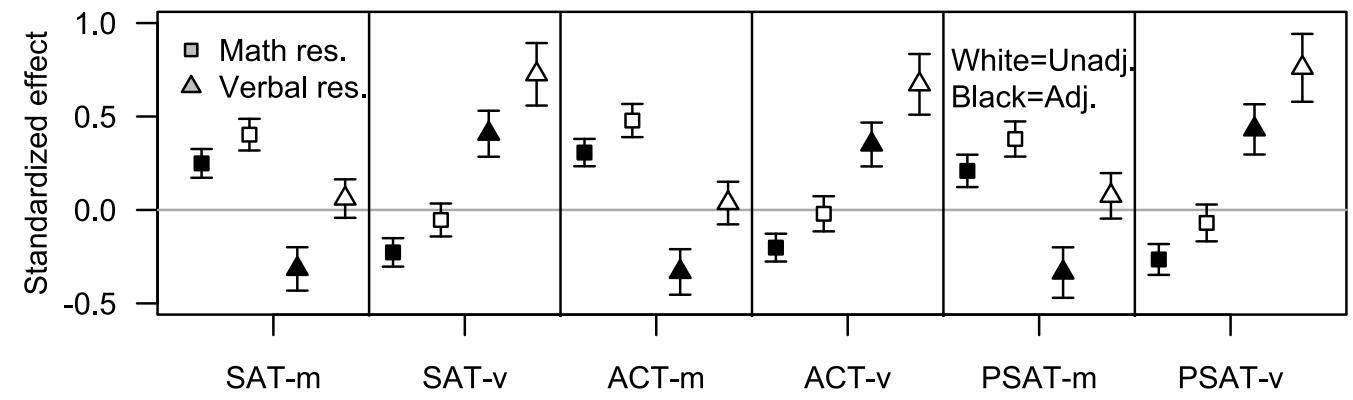

Figure 4. Standardized regression effects of math (squares) and verbal (triangles) non- $g$ residuals on aptitude test scores ( $\mathrm{m}=$ math, $\mathrm{v}=$ verbal) when adjusting (black markers) and when not adjusting (white markers) for $g$. The whiskers indicate $95 \% \mathrm{CI}$. 
association with true math ability rather than a negative association with true verbal non- $g$ residual.

Based on the present findings we offer an alternative explanation of the findings by Coyle and colleagues: As a second order factor, $g$ is defined by the first order factors. Therefore, if a person has the same $g$ as another person but a higher value on one of the defining abilities, e.g. verbal ability, then he/she must have a lower value on at least one of the other abilities, e.g. math ability. Otherwise, they would not have the same value on $g$. If $Y=X+Z$, then $\mathrm{Z}$ must decrease if $\mathrm{X}$ increases in order for $\mathrm{Y}$ to remain constant. Consequently, an observed negative association between the non- $g$ residual on verbal ability and a variable that has a positive association with math ability, e.g. SAT math, when conditioning on $g$, may be confounded by a negative association between the non- $g$ residual on verbal ability and math ability. And vice versa for negative associations between math non- $g$ residuals and variables with a positive association with verbal ability.

In the studies by Coyle and colleagues, the outcome variable has often been regressed on both the non- $g$ residuals on an ability and on the same measure of $g$ that was used to calculate the residuals (Coyle, 2018b; Coyle et al., 2013; Coyle, Snyder, Richmond, et al., 2015; Coyle \& Pillow, 2008). We suspect that this double adjustment for $g$ may be problematic (see Ashton \& Vernon, 1995; Nyborg \& Sommerlund, 1992; Vernon, 1990). It would probably be more reasonable to regress the outcome variable on another measure of $g$ than the one used for calculating the residuals. Ashton and Vernon (1995) found that the partial correlation between verbal and spatial WAIS subtests dropped close to zero, but it did not become negative, when adjusting for $g$ as measured by Raven's Advanced Progressive Matrices. However, the present simulations indicate that if the outcome variable is truly a function of a non- $g$ ability residual, this will be identified by a model where the outcome variable is regressed only on the observed non- $g$ ability residual and not on any measure of $g$ itself and this parameter can, consequently, be dropped altogether.

\section{Limitations}

The present empirical findings are based on data on cognitive abilities and college aptitude tests from one, although relatively large and representative, sample of Americans born between 1980 and 1984 . It is not certain that the main finding, that negative associations between non- $g$ verbal and math ability residuals and aptitude test scores on the other ability may be spurious, generalizes to other populations and other measures of abilities and outcomes.

The present study found that negative associations between non- $g$ residuals on math and verbal ability and aptitude test scores on the other ability ceased to be significant when not double adjusting for $g$. Lack of significant associations could, of course, be due to lack of power in the analyses. However, the power of the study was adequate to identify a significant weakening of the negative associations when not double adjusting for $g$ (no overlap between the CIs of the unadjusted and the corresponding double adjusted negative coefficients in Figure 4). Based on the findings in the simulations, this should warrant the conclusion that the observed negative associations are not due to true associations between the aptitude test scores and the non- $g$ residuals on the other ability.

The present paper should not be interpreted as a denial of the importance of specific abilities. Rather, and more specifically, it is a critique of conclusions that negative $g$-adjusted associations involving non$g$ residuals support theories of the effect of differential investment. On the broader question about the importance of specific abilities we are silent.

\section{Conclusions}

Studies on the effect of non- $g$ ability residuals have often employed double adjustment for $g$, as they have calculated the ability residuals adjusting for $g$ and then calculated the effect of the non- $g$ residuals while adjusting for $g$. Observed negative associations between the non- $g$ residuals on one cognitive ability, e.g. verbal ability, and variables with a positive association with another ability, e.g. SAT math and math ability, may be spurious and due to the double adjustment for $g$ rather than due to differential investment, as suggested in the literature. This conclusion was corroborated by the fact that the associations between non- $g$ residuals on verbal and math ability and aptitude test scores on the other ability vanished when not double adjusting for $g$. Researchers of the effects of specific abilities are recommended to validate their findings and interpretations with analyses not double adjusting for $g$.

\section{References}

Ashton, M. C., \& Vernon, P. A. (1995). Verbal and spatial abilities are uncorrelated when $\mathrm{g}$ is controlled. Personality and Individual Differences, 19(3), 399401. https://doi.org/10.1016/0191-8869(95)00018-2

Calvin, C. M., Fernandes, C., Smith, P., Visscher, P. M., \& Deary, I. J. (2010). Sex, intelligence and educational achievement in a national cohort of over 175,000 11-year-old schoolchildren in England. Intelligence, 38(4), 424-432. https://doi.org/10.1016/j.intell.2010.04.005

Coyle, T. R. (2014). Predictive validity of non-g residuals of tests: More than g. Journal of Intelligence, 2(1), 21-25. https://doi.org/10.3390/jintelligence2010021

Coyle, T. R. (2016). Ability tilt for whites and blacks: Support for differentiation and investment theories. Intelligence, 56, 28-34. https://doi.org/10.1016/j.intell.2016.02.002 
Coyle, T. R. (2019). Tech tilt predicts jobs, college majors, and specific abilities: Support for investment theories. Intelligence, 75, 33-40. https://doi.org/10.1016/j.intell.2019.04.002

Coyle, T. R. (2020). Sex differences in tech tilt: Support for investment theories. Intelligence, 80, 101437. https://doi.org/10.1016/j.intell.2020.101437

Coyle, T. R. (2021). White-Black differences in tech tilt: Support for Spearman's law and investment theories. Intelligence, 84, 101504. https://doi.org/10.1016/j.intell.2020.101504

Coyle, T. R. (2018a). Non-g factors predict educational and occupational criteria: More than g. Journal of Intelligence, 6(3), 43. https://doi.org/10.3390/jintelligence6030043

Coyle, T. R. (2018b). Non-g residuals of group factors predict ability tilt, college majors, and jobs: A non-g nexus. Intelligence, 67, 19-25. https://doi.org/10.1016/j.intell.2017.12.003

Coyle, T. R., \& Greiff, S. (2021). The future of intelligence: The role of specific abilities. Intelligence, 101549. https://doi.org/10.1016/j.intell.2021.101549

Coyle, T. R., \& Pillow, D. R. (2008). SAT and ACT predict college GPA after removing g. Intelligence, 36(6), 719-729. https://doi.org/10.1016/j.intell.2008.05.001

Coyle, T. R., Purcell, J. M., Snyder, A. C., \& Kochunov, P. (2013). Non-g residuals of the SAT and ACT predict specific abilities. Intelligence, 41(2), 114120. https://doi.org/10.1016/j.intell.2012.12.001

Coyle, T. R., Purcell, J. M., Snyder, A. C., \& Richmond, M. C. (2014). Ability tilt on the SAT and ACT predicts specific abilities and college majors. Intelligence, 46, 18-24. https://doi.org/10.1016/j.intell.2014.04.008

Coyle, T. R., Snyder, A. C., \& Richmond, M. C. (2015). Sex differences in ability tilt: Support for investment theory. Intelligence, 50, 209-220. https://doi.org/10.1016/j.intell.2015.04.012

Coyle, T. R., Snyder, A. C., Richmond, M. C., \& Little, M. (2015). SAT non-g residuals predict course specific GPAs: Support for investment theory. Intelligence, 51, 57-66. https://doi.org/10.1016/j.intell.2015.05.003
Gignac, G. E. (2016). Residual group-level factor associations: Possibly negative implications for the mutualism theory of general intelligence.

Intelligence, 55, 69-78. https://doi.org/10.1016/j.intell.2016.01.007

Johnson, W., \& Bouchard, T. J. (2007). Sex differences in mental abilities: $\mathrm{G}$ masks the dimensions on which they lie. Intelligence, 35(1), 23-39. https://doi.org/10.1016/j.intell.2006.03.012

Lynn, R. (1987). The intelligence of the Mongoloids: A psychometric, evolutionary and neurological theory. Personality and Individual Differences, 8(6), 813844. https://doi.org/10.1016/0191-8869(87)90135-8

Nyborg, H., \& Sommerlund, B. (1992). Spearman's g, the verbal-performance balance, and brain processes: The Lynn-Vernon debate. Personality and Individual Differences, 13(11), 1253-1255. https://doi.org/10.1016/0191-8869(92)90263-O

$\mathrm{R}$ Core Team. (2021). $R$ : A language and environment for statistical computing. $R$ Foundation for Statistical Computing, Vienna, Austria. URL https://www.Rproject.org/.

Rosseel, Y. (2012). lavaan: An R package for structural equation modeling. Journal of Statistical Software, 48(2), 1-36. https://www.jstatsoft.org/v48/i02/

Sorjonen, K., Nilsonne, G., Ingre, M., \& Melin, B. (2022). Spurious correlations in research on ability tilt. Personality and Individual Differences, 185, 111268. https://doi.org/10.1016/j.paid.2021.111268

Vernon, P. A. (1990). The effect of holding g constant on the correlation between verbal and nonverbal abilities: A comment on Lynn's "The intelligence of the mongoloids..." (1987). Personality and Individual Differences, 11(7), 751-754. https://doi.org/10.1016/0191-8869(90)90261-O 